\title{
Validation and reclassification of MGAP and GAP in hospital settings using data from the Trauma Audit and Research Network
}

\author{
Rebecca M. Hasler, MD, MSc, Nicole Mealing, PhD, Hans-Ulrich Rothen, MD, Michael Coslovsky, PhD, \\ Fiona Lecky, MD, PhD, and Peter Jüni, MD, Bern, Switzerland
}

BACKGROUND:

METHODS:

RESULTS:

CONCLUSION:

LEVEL OF EVIDENCE: KEY WORDS:

\begin{abstract}
Recently, two simple clinical scores were published to predict survival in trauma patients. Both scores may successfully guide major trauma triage, but neither has been independently validated in a hospital setting.

This is a cohort study with 30-day mortality as the primary outcome to validate two new trauma scores-Mechanism, Glasgow Coma Scale (GCS), Age, and Pressure (MGAP) score and GCS, Age and Pressure (GAP) score - using data from the UK Trauma Audit and Research Network. First, an assessment of discrimination, using the area under the receiver operating characteristic (ROC) curve, and calibration, comparing mortality rates with those originally published, were performed. Second, we calculated sensitivity, specificity, predictive values, and likelihood ratios for prognostic score performance. Third, we propose new cutoffs for the risk categories.

A total of 79,807 adult ( $\geq 16$ years) major trauma patients (2000-2010) were included; 5,474 (6.9\%) died. Mean (SD) age was 51.5 (22.4) years, median GCS score was 15 (interquartile range, 15-15), and median Injury Severity Score (ISS) was 9 (interquartile range, $9-16)$. More than $50 \%$ of the patients had a low-risk GAP or MGAP score ( $1 \%$ mortality). With regard to discrimination, areas under the ROC curve were $87.2 \%$ for GAP score $(95 \%$ confidence interval, $86.7-87.7)$ and $86.8 \%$ for MGAP score (95\% confidence interval, 86.2-87.3). With regard to calibration, 2,390 (3.3\%), 1,900 (28.5\%), and 1,184 (72.2\%) patients died in the low, medium, and high GAP risk categories, respectively. In the low- and medium-risk groups, these were almost double the previously published rates. For MGAP, 1,861 (2.8\%), 1,455 (15.2\%), and 2,158 (58.6\%) patients died in the low-, medium-, and high-risk categories, consonant with results originally published. Reclassifying score point cutoffs improved likelihood ratios, sensitivity and specificity, as well as areas under the ROC curve.

We found both scores to be valid triage tools to stratify emergency department patients, according to their risk of death. MGAP calibrated better, but GAP slightly improved discrimination. The newly proposed cutoffs better differentiate risk classification and may therefore facilitate hospital resource allocation. (J Trauma Acute Care Surg. 2014;77: 757-763. Copyright (C) 2014 by Lippincott Williams \& Wilkins)
\end{abstract}

Prognostic study, level II.

Major trauma; trauma scores; GAP; MGAP; TARN.
$\mathrm{T}$ rauma is the leading cause of death among young people (5-44 years) in developed countries. Worldwide, trauma accounts for $10 \%$ of deaths and $14 \%$ of years of life lost. ${ }^{1,2}$ Accurate prediction of outcomes helps to adequately triage patients according to their risk of death and to make treatment more efficient. ${ }^{3,4}$

Several prognostic scores attempt to accurately predict outcomes for trauma patients. ${ }^{5-10}$ Their complexity limits their usefulness for triage and initial assessment of severity in injured

Submitted: February 24, 2014, Revised: July 29, 2014, Accepted: July 29, 2014 From the Trauma Audit and Research Network (TARN) (R.M.H., F.L.), Salford Royal NHS Foundation Trust, Salford, United Kingdom; and Departments of Emergency Medicine (R.M.H.), and Intensive Care Medicine (H.-U.R.), Inselspital, and Institute of Social and Preventive Medicine and CTU Bern (N.M., M.C., P.J.), University Hospital Bern, Bern, Switzerland.

*R.M.H. and N.M. contributed equally to this study.

The funders did not have any vested interests in the findings of this research and did not have any role in the study design, the data collection, and the writing of the article.

This study was presented at the 14th International Conference on Emergency Medicine (ICEM), in Dublin, Ireland, and Annual Meeting of the Swiss Society of Surgery 2013, in Bern, Switzerland.

Supplemental digital content is available for this article. Direct URL citations appear in the printed text, and links to the digital files are provided in the HTML text of this article on the journal's Web site (www.jtrauma.com).

Address for reprints: Peter Jüni, MD, CTU Bern, Inselspital, Bern University Hospital, Finkenhubelweg 11, 3012 Bern, Switzerland; email: juni@ispm.unibe.ch.

DOI: $10.1097 /$ TA.0000000000000452

I Trauma Acute Care Surg

Volume 77, Number 5 patients in an emergency department setting. Some require laboratory or imaging information, unavailable at initial assessment. Others require clinicians to use complicated formulae, a practice more suited to use in registries. ${ }^{11,12}$ Furthermore, clinical triage scores previously in practical use have tended to ignore the impact of increasing age on the risk of death after injury.

Recently, two succinct clinical scores were developed to predict survival in trauma patients, and they may address some of the problems described earlier. The Mechanism, Glasgow Coma Scale (GCS), ${ }^{13}$ Age and Pressure (MGAP) score ${ }^{14}$ and the GCS, Age and Pressure (GAP) score ${ }^{15}$ derive simple additive scores from age, GCS score, and systolic blood pressure (SBP), which are readily available at the initial assessment. The MGAP also includes the mechanism of trauma (blunt vs. penetrating). ${ }^{14}$ The MGAP score was developed from 360 prehospital trauma patients admitted to mobile intensive care units in France during 2002 and was prospectively validated using patients from the same setting. ${ }^{14}$ The GAP score is based on 27,154 patients from the Japan Trauma Data Bank (2004-2009). ${ }^{15}$ However, the validation sample was representative of the development sample, as the authors randomly divided patients into the two groups. Therefore, excellent validation results were not surprising.

Although both scores may successfully guide major trauma triage, neither has been independently validated in a hospital setting. We therefore set out to validate both scores 
in a large Western population of trauma patients recruited in hospital settings and included in the Trauma Audit and Research Network (TARN). ${ }^{11}$ The hypothesis is that both scores perform well in predicting trauma death at 30 days.

\section{PATIENTS AND METHODS}

TARN is a European multicenter trauma registry with prospective data collection in up to 180 hospitals. It includes all trauma cases admitted directly to a participating hospital and those who require hospitalisation for 72 hours or greater, those who are transferred to a participating hospital for specialist care, those who require a high dependency or intensive care unit, or those who die as a result of their injuries within 93 days. Patients transferred for rehabilitation only; patients with nontraumatic brain injuries, simple skin lacerations, contusions or abrasions, minor penetrating injuries, singular uncomplicated limb injuries; and patients older than 65 years with isolated fractures of the femoral neck or pubic ramus are excluded. ${ }^{16}$ Age, sex, SBP, heart rate, respiratory rate, GCS score, mechanism of injury, and the Injury Severity Score (ISS) are all recorded. ${ }^{16,17}$ Research ethics permission for research on the anonymized data held by TARN was granted by the National Information Governance Board (approval number: PIAG3-04(e)/2006).

For inclusion in our analysis, TARN patients had to be 16 years or older and admitted to a participating hospital in the United Kingdom between January 2000 and December 2010. Patients from continental European hospitals were excluded because trauma care varies across continental European countries. We also excluded patients if they had been transferred from a non-TARN hospital, since data on their clinical status at admission was missing; if they were referred to a non-TARN hospital, because information on mortality was missing; or if data on any of the four components used to derive the GAP or MGAP scores was missing (age, SBP at arrival at the emergency department, GCS score, and mechanism of trauma).

The GAP score ranges from 3 to 24 ; total score is composed of the actual GCS value (3-15 points), plus 3 points if age at admission is less than 60 years, 4 points if SBP is greater than $60 \mathrm{~mm} \mathrm{Hg}$ to $120 \mathrm{~mm} \mathrm{Hg}$, and 6 points if SBP is greater than $120 \mathrm{~mm} \mathrm{Hg}$. Higher values indicate better prognosis (Table 1). ${ }^{15}$ Scores from 3 to 10 indicate high, 11 to 18 moderate, and 19 to 24 low risk of death up to 30 days. $^{15}$ The MGAP ranges from 3 to 29; total score is composed of the actual GCS value (3-15 points), plus 5 points if age at

TABLE 1. Comparison of GAP and MGAP Score Point Allocations

\begin{tabular}{lcc}
\hline Score Component & $\begin{array}{c}\text { GAP Score } \\
\text { (Point Range, 3-24) }\end{array}$ & $\begin{array}{c}\text { MGAP Score } \\
\text { (Point Range, 3-29) }\end{array}$ \\
\hline Age $<60$ y & +3 & +5 \\
SBP $>120 \mathrm{~mm} \mathrm{Hg}$ & +6 & +5 \\
SBP of $60-120 \mathrm{~mm} \mathrm{Hg}$ & +4 & +3 \\
GCS score & GCS value & GCS value \\
Blunt trauma & - & +4 \\
$\quad$ vs. penetrating) & & \\
\hline \multicolumn{2}{c}{ GCS score ranges from 3 points to 15 points. }
\end{tabular}

GCS score ranges from 3 points to 15 points. admission is less than 60 years, 3 points if SBP is greater than $60 \mathrm{~mm} \mathrm{Hg}$ to $120 \mathrm{~mm} \mathrm{Hg}, 5$ points if SBP is greater than $120 \mathrm{~mm} \mathrm{Hg}$, and 4 points if the patient experienced blunt trauma. Scores from 3 to 17 indicate high, 18 to 22 moderate, and 23 to 29 low risk of death up to 30 days (Table 1). ${ }^{14}$

In this cohort study, the primary outcome was overall mortality up to 30 days. To assess discrimination, ${ }^{18}$ we used a logistic regression model to estimate the area under the receiver operating characteristic curve (ROC) for 30-day mortality. An area under the ROC of 1 is optimal, and an area of 0.5 indicates that the score is no better than chance at assessing the risk of death. ${ }^{19}$ We plotted Kaplan-Meier cumulative incidence curves by originally proposed risk categories and calculated corresponding hazard ratios (HRs) using Cox models with shared frailty for hospitals to account for the clustering of patients within hospitals. ${ }^{20}$ We used the low-risk group as the reference group. To determine calibration, ${ }^{18}$ we calculated mortality rates by risk category and compared them with mortality rates previously published by the groups who developed the scores. ${ }^{14,15}$ Then, we used univariable Cox models with shared frailty for hospitals to determine the association of patient characteristics with mortality.

To assess the prognostic performance, without regard to originally specified cutoffs, we calculated sensitivity, specificity, positive predictive value, negative predictive value, as well as positive and negative likelihood ratios (LRs). We used each individual score point as a cutoff for dichotomizing patients into categories of higher risk of death (less than or equal to the cutoff) and lower risk of death (greater than the cutoff). Positive LR indicates how much more likely it is to find scores less than or equal to the cutoff in patients who died than in patients who survived. Conversely, negative LR specifies how much less likely it is to find scores greater than the cutoff in patients who died than in patients who survived.

We propose new cutoffs for the categorization of patients into low, moderate, and high risk of mortality based on the calculated LR. The cutoff for the high-risk category was set at the maximum score at which the positive LR was 5 or greater (corresponding to moderate power to rule in patients at high risk of death); ${ }^{21}$ the cutoff for the low-risk category was set at the minimum score at which the negative LR was 0.1 or lower (corresponding to a high power to rule out patients at moderate to high risk of death) ${ }^{21}$ Patients with scores between the cutoffs were at moderate risk. Finally, we determined the numbers of patients classified into low-, moderate-, and high-risk categories and the corresponding numbers of deaths observed within these categories. We used the cutoffs originally published by Kondo et al. ${ }^{15}$ and Sartorius et al. ${ }^{14}$ and the new cutoffs for a hypothetical population of 1,000 trauma patients presenting to a major trauma center. To account for missing data, we performed a sensitivity analysis using multiple imputation with mortality, survival time and age, sex, SBP, GCS score, ISS, mechanism of injury, and hospital as variables in the imputation model. All analyses were completed using Stata, Release 11 (StataCorp LP, College Station, TX).

\section{RESULTS}

From 2000 to 2010, 138,097 patients from 180 hospitals were included in the registry. A total of 79,807 TARN patients 
$(57.8 \%)$ matched the inclusion criteria; patients from non-UK hospitals $(n=6,952)$, those younger than 16 years $(n=13,389)$, those transferred from or to a non-TARN hospital $(n=28,250)$, those with missing data for any of the four score component variables $(n=9,682)$, or those who died but had no or an invalid date of death recorded were excluded ( $n=17$, Fig. 1 ). Patient characteristics and their univariable associations with 30 -day mortality are shown in Table 2 . A total of 5,474 (6.9\%) died within 30 days. The mean (SD) age was 51.5 (22.4) years, the mean SBP upon arrival at ED was 135 (30.1) $\mathrm{mm} \mathrm{Hg}$, the median GCS score was 15 (interquartile range, 15-15), and the median ISS was 9 (interquartile range, 9-16). Additional patient characteristics are illustrated in Supplementary Table 1 (http://links.lww.com/TA/A478). Supplementary Figure 1 (http://links.lww.com/TA/A479) shows the distribution of GAP and MGAP score points and their association with 30-day mortality. More than half of the patients had a GAP score of 22 or greater, which was associated with a $1 \%$ risk of death, and more

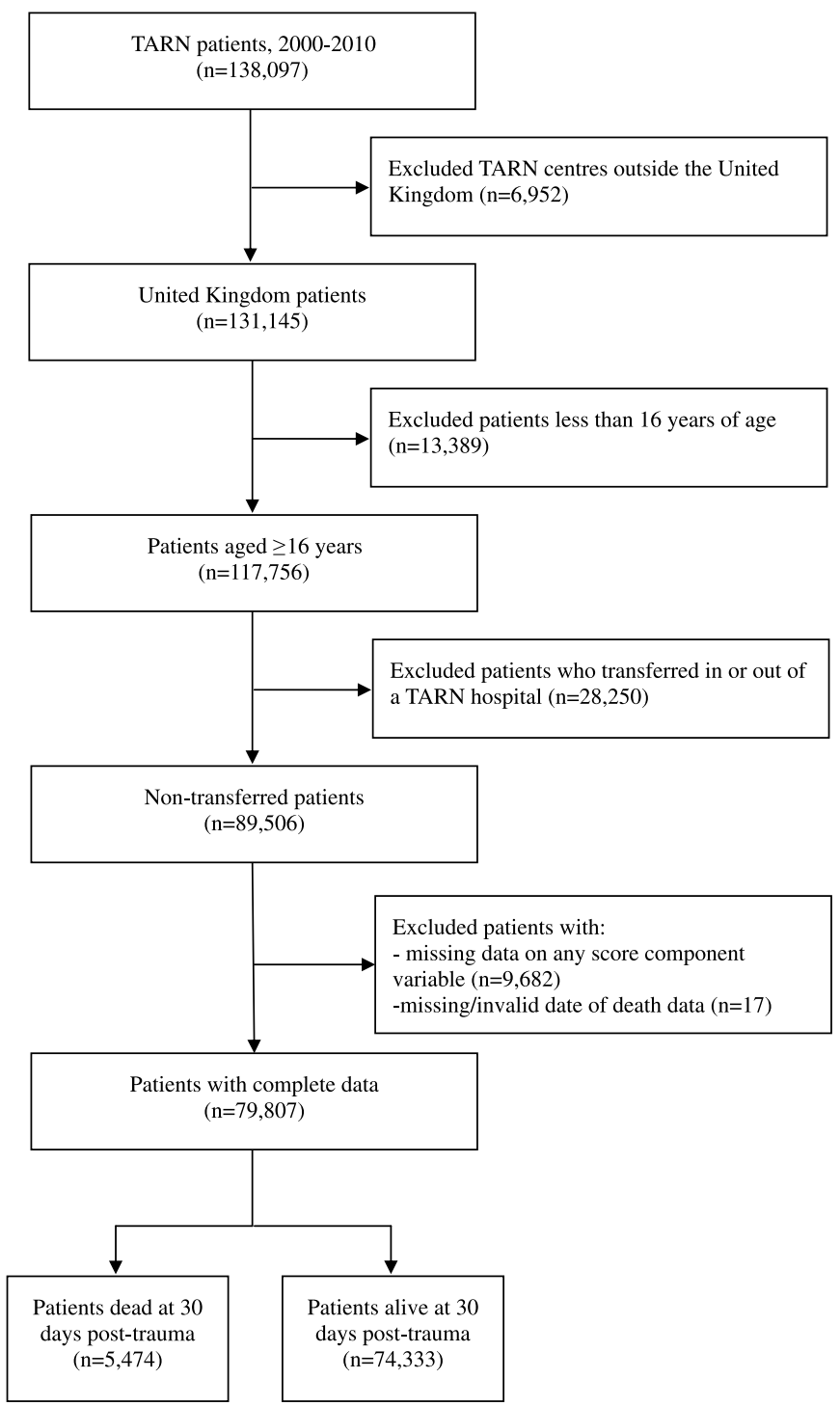

Figure 1. Flow diagram of included and excluded patients.
TABLE 2. Baseline Characteristics of TARN Patients With Crude HRs Within 30 Days $(n=79,807)$

\begin{tabular}{|c|c|c|c|c|}
\hline \multirow[b]{2}{*}{ Characteristics } & \multicolumn{4}{|c|}{ Mortality Within 30 d After Trauma } \\
\hline & $\begin{array}{l}\text { Yes, n (\%) } \\
(\mathrm{n}=5,474)\end{array}$ & $\begin{array}{c}\text { No, } n(\%) \\
(\mathrm{n}=74,333)\end{array}$ & $\begin{array}{c}\text { HR* }^{*} \\
(95 \% \text { CI })\end{array}$ & $p$ \\
\hline \multicolumn{5}{|l|}{ Age, y } \\
\hline $16-30$ & 800 (14.6) & $18,248(24.5)$ & 1.00 (reference) & $<0.001$ \\
\hline $31-45$ & $633(11.6)$ & $15,103(20.3)$ & $0.97(0.88-1.08)$ & \\
\hline $46-60$ & $694(12.7)$ & $16,218(21.8)$ & $1.05(0.95-1.17)$ & \\
\hline $61-75$ & $952(17.4)$ & $12,025(16.2)$ & $1.97(1.79-2.16)$ & \\
\hline $76-110$ & $2,395(43.8)$ & $12,739(17.1)$ & $4.42(4.07-4.80)$ & \\
\hline \multicolumn{5}{|l|}{ Sex } \\
\hline Male & $3,334(60.9)$ & $46,397(62.4)$ & 1.00 (reference) & 0.0065 \\
\hline Female & $2,140(39.1)$ & $27,936(37.6)$ & $1.08(1.02-1.14)$ & \\
\hline \multicolumn{5}{|l|}{ SBP, mm Hg } \\
\hline $0-59$ & $213(3.9)$ & $214(0.3)$ & $17.9(15.4-20.8)$ & $<0.001$ \\
\hline $60-79$ & $307(5.6)$ & $731(1.0)$ & $8.25(7.26-9.38)$ & \\
\hline $80-99$ & $577(10.5)$ & $3,435(4.6)$ & $3.63(3.28-4.02)$ & \\
\hline $100-119$ & $827(15.1)$ & $13,148(17.7)$ & $1.44(1.31-1.58)$ & \\
\hline $120-139$ & $1,040(19.0)$ & $23,968(32.2)$ & 1.00 (reference) & \\
\hline $140-159$ & $1,094(20.0)$ & $19,911(26.8)$ & $1.26(1.16-1.37)$ & \\
\hline $160-179$ & $656(12.0)$ & $8,194(11.0)$ & $1.81(1.64-1.99)$ & \\
\hline $180-199$ & $452(8.3)$ & $3,322(4.5)$ & $2.98(2.67-3.33)$ & \\
\hline $200-250$ & $308(5.6)$ & $1,410(1.9)$ & $4.55(4.00-5.17)$ & \\
\hline \multicolumn{5}{|l|}{ GCS score } \\
\hline 3 & $1,462(26.7)$ & $946(1.3)$ & $31.0(29.0-33.2)$ & $<0.001$ \\
\hline $4-5$ & $462(8.4)$ & $548(0.7)$ & $19.4(17.5-21.5)$ & \\
\hline $6-8$ & $509(9.3)$ & $1,603(2.2)$ & $8.47(7.68-9.33)$ & \\
\hline $9-12$ & $572(10.4)$ & $2,687(3.6)$ & $5.77(5.26-6.32)$ & \\
\hline $13-15$ & $2,469(45.1)$ & $68,549(92.2)$ & 1.00 (reference) & \\
\hline \multicolumn{5}{|c|}{ 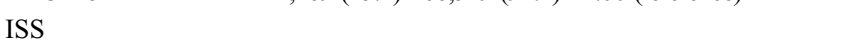 } \\
\hline $1-8$ & $368(6.7)$ & $18,512(24.9)$ & 1.00 (reference) & $<0.001$ \\
\hline $9-15$ & $1,075(19.6)$ & $38,630(52.0)$ & $1.40(1.24-1.57)$ & \\
\hline $16-25$ & $1,740(31.8)$ & $12,422(16.7)$ & $7.19(6.42-8.05)$ & \\
\hline $26-75$ & $2,291(41.9)$ & $4,769(6.4)$ & $23.2(20.8-26.0)$ & \\
\hline \multicolumn{5}{|l|}{ Mechanism of Injury } \\
\hline Blunt & $5,303(96.9)$ & $70,554(94.9)$ & 1.00 (reference) & $<0.001$ \\
\hline Penetrating & $171(3.1)$ & $3,779(5.1)$ & $0.59(0.51-0.69)$ & \\
\hline \multicolumn{5}{|l|}{ GAP, 3 risk categories } \\
\hline Low, 19-24 & $2,390(43.7)$ & $69,100(93.0)$ & 1.00 (reference) & $<0.001$ \\
\hline Medium, 11-18 & $1,900(34.7)$ & $4,778(6.4)$ & $10.3(9.71-11.0)$ & \\
\hline High, 3-10 & $1,184(21.6)$ & $455(0.6)$ & $43.2(40.2-46.4)$ & \\
\hline \multicolumn{5}{|c|}{ MGAP, 3 risk categories } \\
\hline Low, 23-29 & $1,861(34.0)$ & $64,715(87.1)$ & 1.00 (reference) & $<0.001$ \\
\hline Medium, $18-22$ & $1,455(26.6)$ & $8,092(10.9)$ & $5.81(5.42-6.22)$ & \\
\hline High, 3-17 & $2,158(39.4)$ & $1,526(2.1)$ & $33.9(31.8-36.2)$ & \\
\hline
\end{tabular}

*HRs are from Cox regression models with shared frailty for hospitals to account for clustering of patients within hospitals. HRs larger than 1 indicate a higher mortality than in the reference group. $p$ values are from tests for linear trend or association.

than half of the patients had a MGAP score of 25 or greater, which was associated with a $1 \%$ risk of death.

Figure 2 presents areas under the ROC curve of $87.2 \%$ for GAP scores (95\% confidence interval [CI], 86.7-87.7\%, Fig. $2 A)$, and $86.8 \%$ for MGAP scores $(95 \% \mathrm{CI}, 86.2-87.3 \%$, Fig. $2 B$ ). Using the originally proposed cutoffs to derive risk categories, ${ }^{14,15}$ rather than the full scores, we found areas of $86.7 \%$ 


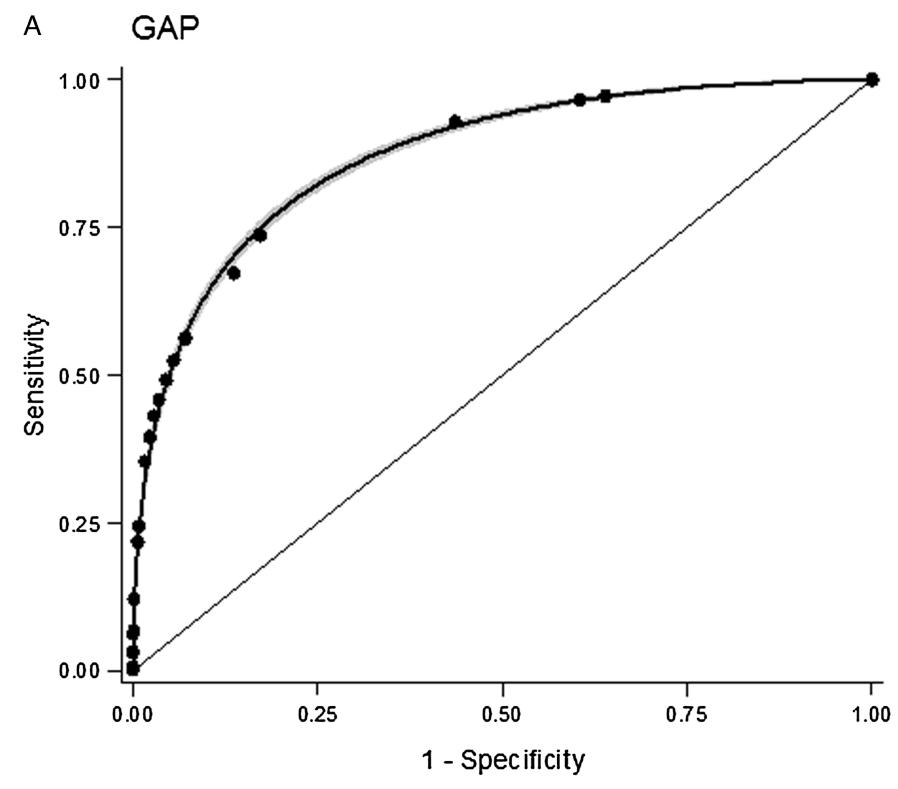

Area under curve $=0.872(95 \% \mathrm{Cl}: 0.867-0.877)$

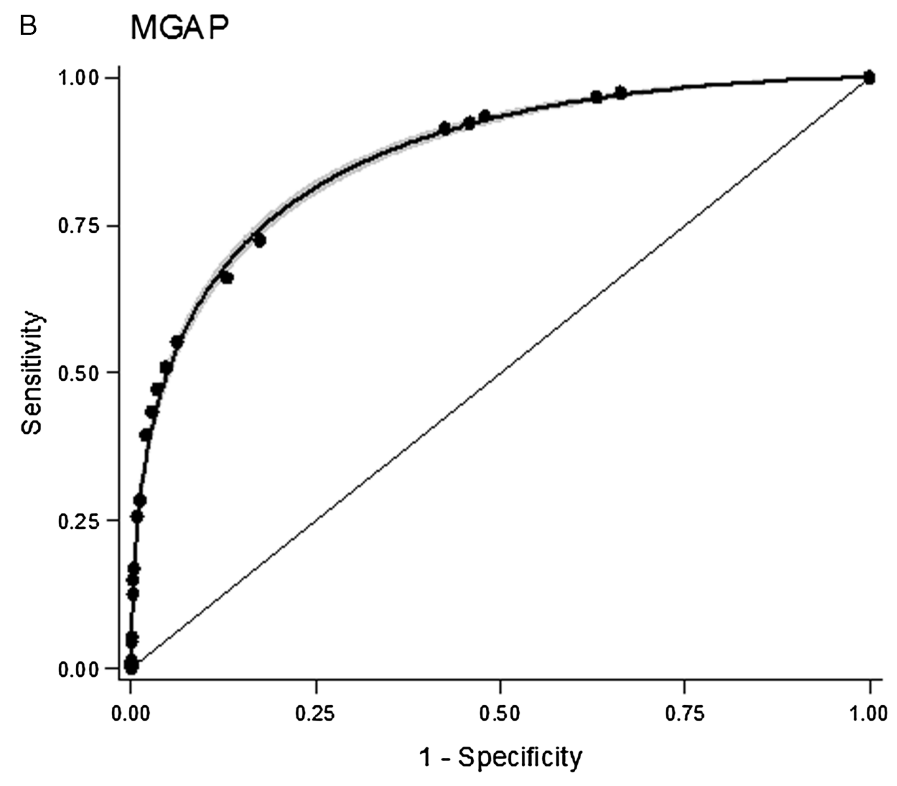

Area under curve $=0.868(95 \% \mathrm{Cl}: 0.862-0.873)$

Figure 2. Figure 2 presents areas under the ROC curve for GAP and for MGAP scores using the originally proposed cut-offs to derive risk categories.

(95\% CI, 85.8-87.6\%) and 84.3\% (95\% CI, 83.4-85.2\%). Supplementary Figure 2 (http://links.lww.com/TA/A480) presents Kaplan-Meier curves by risk category for GAP (left) and MGAP (right). According to GAP, corresponding HRs of death up to 30 days were $10.3(95 \% \mathrm{CI}, 9.7-11.0)$ for patients at moderate risk according to GAP and 43.2 (95\% CI, 40.1-46.4) for patients at high risk compared with patients with low risk ( $p$ for trend $<0.001)$. Corresponding HRs for MGAP were $5.8(95 \% \mathrm{CI}$, 5.4-6.2) and 33.9 (95\% CI, 31.8-36.2; $p$ for trend $<0.001)$.

Figure 3 presents observed mortality rates by risk category and compares them to previously published mortality rates. ${ }^{14,15} \mathrm{~A}$ total of 2,390 (3.3\%, 95\% CI, 3.2-3.5\%), 1,900 $(28.5 \%, 27.4-29.5 \%)$, and $1,184(72.2 \%, 70.1-74.4 \%)$ patients died in the low, medium, and high GAP risk categories, respectively. In low- and medium-risk groups, these estimates were almost double the previously published rates of $1.8 \%$ $(1.5-2.1 \%)$ and $14.9 \%(13.3-16.5 \%) .{ }^{15}$ In the low, medium, and high MGAP risk categories, respectively, 1,861 (2.8\%,
2.7-2.9\%), 1,455 (15.2\%, 14.5-16.0\%), and 2,158 (58.6\%, $57.0-60.2 \%$ ) patients died. These rates were similar to those previously published (Fig. 5)..$^{14}$

Supplementary Table 2 (http://links.lww.com/TA/A481) presents corresponding estimates of GAP performance according to different cutoffs. At a cutoff of 18 or lower, the score reached a clinically relevant power to rule in patients at risk of death, with a positive LR of $8.00(95 \%$ CI, 7.73-8.29). At this cutoff, sensitivity and specificity were $56.3 \%$ (55.0-57.6\%) and $93.0 \%$ (92.8-93.1\%), respectively. At a cutoff of 21 or greater, the GAP reached a high power to rule out patients at risk of death, with a negative LR of $0.09(0.08-0.10)$ and sensitivity and specificity of $96.6 \%(96.0-97.0 \%)$ and $39.4 \%$ (39.1-39.8\%), respectively. Using these cutoffs to derive new risk categories, we found 3,084 deaths in the high-risk category of 3 points to 18 points $(37.1 \%, 36.0-38.1 \%), 2,003$ in the moderate-risk category of 19 points to 21 points $(6.8 \%$, $6.5-7.1 \%$ ), and 387 in the low-risk category of 22 points to
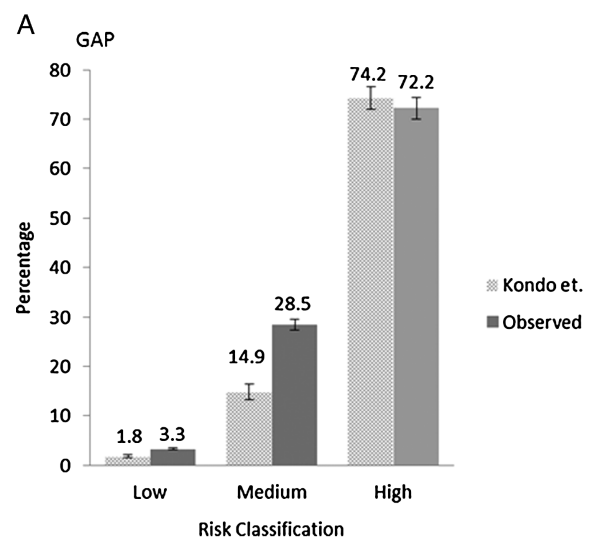

Figure 3.

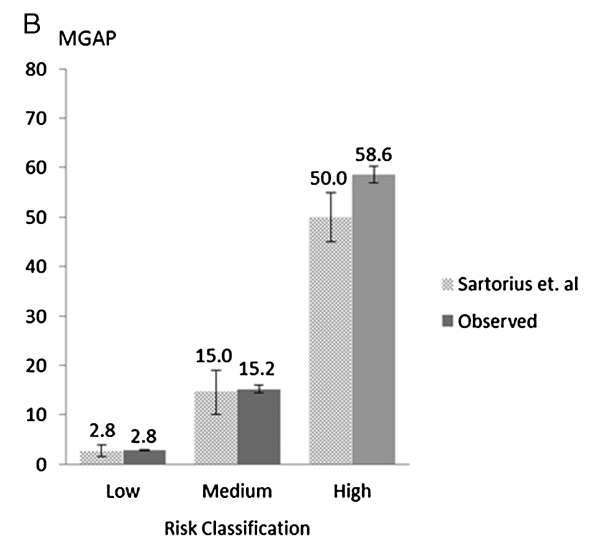


24 points $(0.9 \%, 0.8-1.0 \%)$. The area under the ROC curve was $87.6 \%$ (95\% CI, 87.0-88.1\%).

Supplementary Table 3 (http://links.lww.com/TA/A482) presents corresponding estimates of the performance of the MGAP according to different cutoffs. At a cutoff of 22 or lower, the score reached a clinically relevant power to rule in patients at risk of death, with a positive LR of 5.10 (95\% CI, 4.97-5.24). At this cutoff, sensitivity and specificity were $66.0 \%(64.7-67.2 \%)$ and $87.1 \%(86.8-87.3 \%)$, respectively. At a cutoff of 27 or greater, the MGAP reached a high power to rule out patients at risk of death, with a negative LR of $0.09(0.08-0.10)$ and sensitivity and specificity of $96.8 \%$ (96.3-97.2\%) and 36.9\% (36.5-37.2\%), respectively. Using these cutoffs to derive new risk categories, we found 3,613 deaths in the high-risk category of 3 points to 22 points $(27.3 \%$, $26.5-28.1 \%), 1,500$ in the moderate-risk category of 23 points to 26 points $(5.5 \%, 5.2-5.7 \%)$, and 361 in the low-risk category of 27 points to 29 points $(0.9 \%, 0.8-1.0 \%)$. The area under the ROC curve was $86.3 \%$ (95\% CI, 85.8-86.8\%).

Figure 4 graphically presents the numbers of patients classified into low-, moderate-, and high-risk categories and the corresponding numbers of deaths observed within these categories using the cutoffs originally published (top) and the new cutoffs (bottom) for a hypothetical population of 1,000 trauma patients presenting to a major trauma center for GAP (left) and MGAP (right). For both scores, according to the new categorization, the original medium- and high-risk categories

A

GAP, original categorisation

Low: 19-24

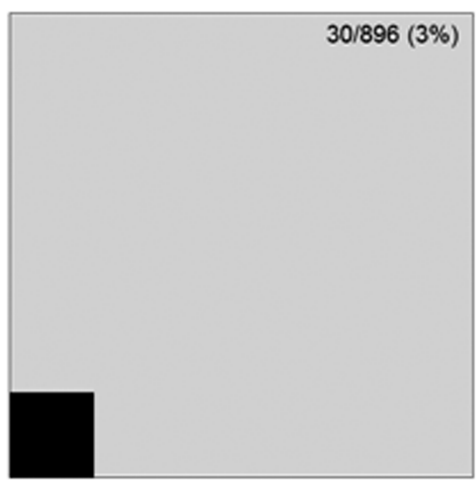

Moderate: $11-18$

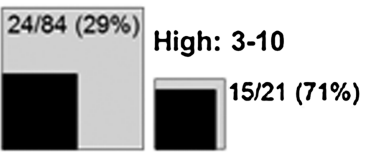

C

GAP, revised categorisation

Low: 22-24

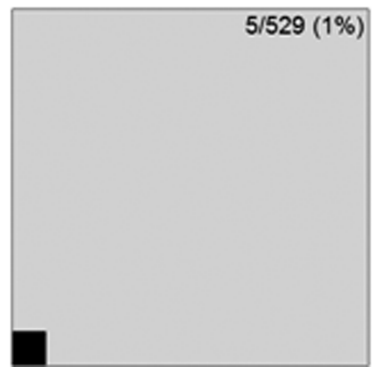

Moderate: $19-21$

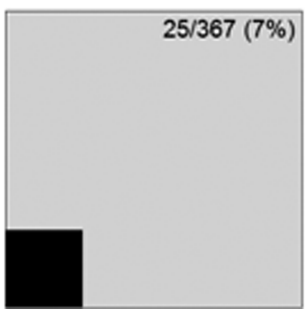

High: $3-18$

$39 / 104(38 \%)$

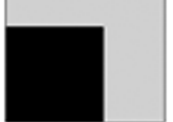

were collapsed into the same category and labelled as high risk. Conversely, the original low-risk categories were split into lowand moderate-risk categories. For both scores, the new low-risk category contained only 5 of a total of 69 hypothetical deaths occurring in the overall population of 1,000 patients, but the high-risk category contained 39 and 45 deaths.

In a sensitivity analysis using multiple imputation to account for missing data and additional inclusion of the 9,682 patients with missing components for GAP or MGAP, the area under the ROC curve remained unchanged $(87.5 \%$ for GAP, $87.2 \%$ for MGAP). Predictive values, sensitivity, specificity, and LRs as reported in the Supplementary Tables 5 and 6 (http://links.lww.com/TA/A483 and http://links.lww.com/TA/A484) were compatible with those found in the main analyses.

\section{DISCUSSION}

Both MGAP and GAP performed well in predicting 30-day mortality, with similar areas under the ROC curve (approximately 87\%) in our independent validation of GAP and MGAP in 79,807 trauma patients from the TARN registry. Calibration was better for MGAP, while GAP was slightly better at discriminating with both original and novel cutoffs. For both scores, our novel cutoffs for risk categories improved differentiation of patients. Approximately $50 \%$ of patients were classified as low risk, of whom only approximately $1 \%$ died. Of

B MGAP, original categorisation

Low: $23-29$

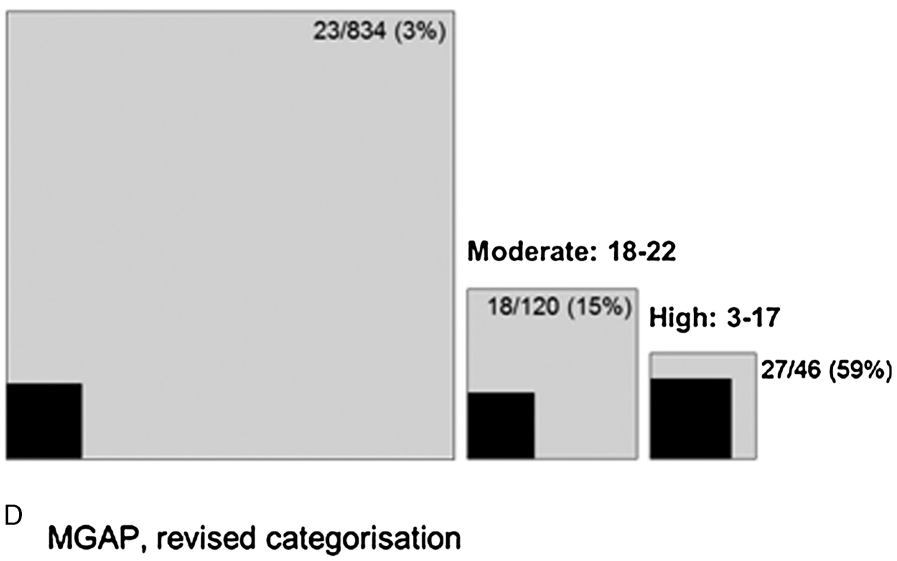

Low: 27-29

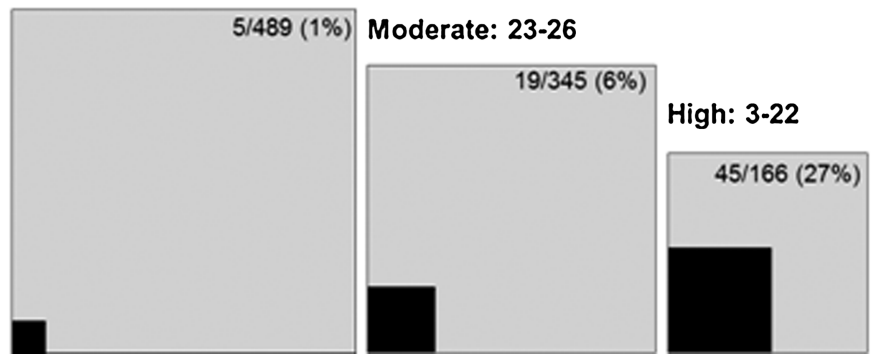

Figure 4. 
the patients, $10 \%$ to $15 \%$ were classified as high risk; one quarter to one third of them died.

This study was validated with a very large sample of UK major trauma patients with prospective data collection from 180 hospitals. Because patient characteristics may vary by hospital, we used Cox models with shared frailty for hospitals to account for the hierarchical structure in the data set. For both scores, sensitivity, specificity, positive predictive value, negative predictive value, and LRs were calculated for different cutoffs, and this allowed us to better differentiate in terms of patient classification.

This analysis was register based, and we were therefore limited by missing data in some covariates, which is a wellknown problem for registry studies. In this study, either automated oscillometry or manual sphygmomanometry were used for SBP measurement because this represents the current practice in emergency departments. However, this might have led to random misclassification of some patients. We relied on clinical data collected at patient admission to participating emergency departments. Thus, our results might not be generalizable to prehospital settings.

The MGAP score was developed from 360 prehospital trauma patients admitted to mobile intensive care units in France during 2002 and was prospectively validated using a sample of 1,003 patients from the same setting from 2003 to 2005. ${ }^{14}$ Although this score was designed for use in a prehospital setting, our study demonstrated its validity for patients admitted to emergency departments. The GAP score is based on data collected on 27,154 patients from the Japan Trauma Data Bank, between 2004 and 2009. The authors randomly divided these patients into a development sample of 13,463 patients and a validation sample of 13,691 patients. ${ }^{15}$ The validation sample was representative of the development sample, so it is not surprising that it yielded excellent results. Our independent validation included considerably more patients than either of the two studies. We found that both scores perform well in an in-hospital setting for triage of patients admitted with major trauma to emergency departments.

MGAP differentiates between blunt and penetrating injury mechanism, allocating lower values — and therefore higher risk - to patients with penetrating trauma. ${ }^{14}$ However, the univariate analysis for mechanism of trauma shows that penetrating trauma is associated with a lower risk of death than is blunt trauma (HR for penetrating trauma patients, $0.59 ; 95 \% \mathrm{CI}$, 0.51-0.69). In view of the small number of patients who experienced penetrating trauma, this should not have a large impaction the analysis.

In addition to age and GCS score, known for their correlation with mortality in trauma patients, ${ }^{22}$ both scores also include an upper cutoff for SBP at $120 \mathrm{~mm} \mathrm{Hg}$. This is a slightly higher cutoff than we found in our previous work on hypotension in blunt and penetrating trauma patients, in which patients with an SBP of less than $110 \mathrm{~mm} \mathrm{Hg}$ were found to be at increased risk of death. ${ }^{23,24}$ However, these previous studies excluded patients with head trauma, and so a slightly higher cutoff of SBP in the general trauma population is reasonable. As shown by a study by Perel et al., ${ }^{25}$ cutoffs for vital signs such as SBP as well as age and GCS may also vary between countries.
Cutoffs originally defined by GAP and MGAP to classify patients were based on average risk of death, distinguishing between a low-risk group ( $<5 \%$ ), a moderate-risk group (5-50\%), and a high-risk group ( $>50 \%$ mortality). These cutoffs classified fewer than $5 \%$ of patients as high risk, and approximately two of three patients died. More than $80 \%$ were classified as low risk, and approximately $3 \%$ died. To derive our alternate cutoffs, we focused on the power to rule in or out patients at high risk of death, assessed by LRs instead of average mortality risks. Our classification is more conservative and better differentiated patients with low mortality risk, while the persistently high area under the ROC curve indicated that its ability to discriminate was not diminished.

GAP and MGAP are new and simple tools for the triage of patients with major trauma in the in-hospital setting. A valid and accurate risk assessment is likely to positively influence the management of these patients. According to the newly proposed cutoffs, $10 \%$ to $15 \%$ of patients were classified as high risk and one of three patients died, whereas approximately $50 \%$ of patients were classified as low risk and approximately $1 \%$ died. The low mortality risk means that patients could be safely allocated to regular management. High-risk patients could be directly referred to the resuscitation room and receive specific care from a trauma team, which includes emergency physicians, surgeons, and anaesthesiologists. Moderate-risk patients could be considered for referral to specific trauma care with regular observation of vital signs and clinical status of the patient. At a major European trauma center with approximately 500 TARN patients per year, this would implicate that less than one patient per day would be triaged as moderate or high risk and therefore increased use of resuscitation rooms may not be a problem applying the reclassified MGAP or GAP scores. Kaplan-Meier curves in Supplementary Figure 2 (http://links.lww.com/TA/A480) indicate that most deaths occur within 24 hours to 48 hours. Therefore, our conclusions would remain identical if short-term mortality had been addressed in our analysis rather than death at 30 days.

There is some evidence that the power of the GCS can be adequately represented by the best motor response segment. ${ }^{26}$ However, Vivien et al. ${ }^{27}$ found a subtle increase in mortality rate in the low-risk stratum for MGAP, replacing the GCS by its motor component. Therefore, studying the two score models by using just best motor response only would be a topic for further research.

\section{CONCLUSION}

In conclusion, we found the GAP and MGAP scores to be valid triage tools for use in in-hospital settings to stratify patients with major trauma according to their risk of death. The newly proposed cutoffs result in a more differentiated risk classification and could allow more informed resource allocation.

\section{AUTHOSHIP}

R.M.H. and P.J. conceived the study. P.J., R.M.H. and N.M. were responsible for the conception and design of the study. P.J., R.M.H., F.L., M.C. and N.M. were responsible for the data analysis and interpretation in collaboration with H.-U.R. F.L. and the Trauma Audit and Research Network were responsible for the acquisition of the data. R.M.H. and 
N.M. wrote the first draft of the manuscript. All authors critically revised the manuscript for important intellectual content and approved the final version of the manuscript.

\section{ACKNOWLEDGMENT}

We thank Prof. Dr. Aristomenis K. Exadaktylos for his support in the clinical questions and for proof reading the manuscript.

We thank Thomas Jenks for the data set preparation as well as to the other staff of the TARN.

We also thank all TARN hospitals for their data contribution.

\section{DISCLOSURE}

R.M.H. has been supported by the Fürstlicher Kommerzienrat Guido Feger Foundation and the Gottfried und Julia Bangerter-Rhyner Foundation. The authors state that no fundings from National Institutes of Health $(\mathrm{NIH})$, Wellcome Trust, and the Howard Hughes Medical Institute (HHMI) have been received. The authors declare that they have no financial or nonfinancial conflicts of interest.

\section{REFERENCES}

1. World Health Organisation (WHO). World Health Statistics 2009: Cause Specific Mortality and Morbidity. Available at: http://www.who.int/ whosis/whostat/EN_WHS09_Table2.pdf. Accessed January 18, 2014.

2. Krug EG, Sharma GK, Lozano R. The global burden of injuries. Am J Public Health. 2000;90:523-526.

3. Hasler RM, Kehl C, Exadaktylos AK, Albrecht R, Dubler S, Greif R, Urwyler N. Accuracy of prehospital diagnosis and triage of a Swiss helicopter emergency medical service. J Trauma Acute Care Surg. 2012; 73(3):709-715.

4. Centers for Disease Control and Prevention. Morbidity and Mortality Weekly Report. January 13, 2012. Guidelines for Field Triage of Injured Patients. Recommendations of the National Expert Panel on Field Triage 2011. Available at: http://www.cdc.gov/mmwr/pdf/rr/rr6101.pdf. Accessed January 18, 2014.

5. Bouamra O, Wrotchford A, Hollis S, Vail A, Woodford M, Lecky F. A new approach to outcome prediction in trauma: a comparison with the TRISS model. $J$ Trauma. 2006;61(3):701-710.

6. Willis CD, Gabbe BJ, Jolley D, Harrison JE, Cameron PA. Predicting trauma patient mortality: ICD [or ICD-10-AM] versus AIS based approaches. ANZ J Surg. 2010;80(11):802-806.

7. Raum MR, Nijsten MW, Vogelzang M, Schuring F, Lefering R, Bouillon B, Rixen D, Neugebauer EA, Ten Duis HJ. Polytrauma Study Group of the German Trauma Society. Emergency trauma score: an instrument for early estimation of trauma severity. Crit Care Med. 2009;37(6):1972-1977.

8. Kuhls DA, Malone DL, McCarter RJ, Napolitano LM. Predictors of mortality in adult trauma patients: the physiologic trauma score is equivalent to the Trauma and Injury Severity. J Am Coll Surg. 2002;194(6):695-704.

9. Panczykowski DM, Puccio AM, Scruggs BJ, Bauer JS, Hricik AJ, Beers SR, Okonkwo DO. Prospective independent validation of IMPACT modeling as a prognostic tool in severe traumatic brain injury. $J$ Neurotrauma. 2012;29(1):47-52.

10. Huber-Wagner S, Stegmaier J, Mathonia P, Paffrath T, Euler E, Mutschler W, Kanz KG, Lefering R, Working Group on Polytrauma (NIS) of the German Trauma Society (DGU). The sequential trauma score-a new instrument for the sequential mortality prediction in major trauma. Eur $J$ Med Res. 2010;15(5):185-195.

11. The Trauma Audit and Research Network (TARN). Available at: https:// www.tarn.ac.uk/Login.aspx. Accessed January 18, 2014.

12. Raum MR. Validating scores: a good business for a trauma register. Crit Care. 2012;16(5):159.

13. Teasdale G, Jennett B. Assessment of coma and impaired consciousness. A practical scale. Lancet. 1974;2(7872):81-84.

14. Sartorius D, Le Manach Y, David JS, Rancurel E, Smail N, Thicoipé M, Wiel E, Ricard-Hibon A, Berthier F, Gueugniaud PY, et al. Mechanism, Glasgow Coma Scale, Age, and Arterial Pressure (MGAP): a new simple prehospital triage score to predict mortality in trauma patients. Crit Care Med. 2010;38(3):831-837.

15. Kondo Y, Abe T, Kohshi K, Tokuda Y, Cook EF, Kukita I, Revised trauma scoring system to predict in-hospital mortality in the emergency department: Glasgow Coma Scale, age and systolic blood pressure score. Crit Care. 2011;15(4):R191.

16. The Trauma Audit and Research Network (TARN), Procedures Manual February 2013. Available at: https://www.tarn.ac.uk/content/downloads/ 53/Full\%20Procedures\%20manual\%20-\%20February\%202013.pdf. Accessed January 18, 2014.

17. Committee on Injury Scaling. Association for the Advancement of Automotive Medicine (AAAM). The Abbreviated Injury Scale 2008 Revision. Des Plaines, IL: AAAM; 2008.

18. McGilchrist CA, Aisbett CW. Regression with frailty in survival analysis. Biometrics. 1991;47:461-466.

19. Jaeschke R, Guyatt GH, Sackett DL; The Evidence-Based Medicine Working Group. Users' guides to the medical literature. III. How to use an article about a diagnostic test. B. What are the results and will they help me in caring for my patients? JAMA. 1994;271(9):703-707.

20. Justice AC, Covinsky KE, Berlin JA. Assessing the generalizability of prognostic information. Ann Intern Med. 1999;130(6):515-524.

21. Hanley JA, McNeil BJ. The meaning and use of the area under a receiver operating characteristic (ROC) curve. Radiology. 1982;143:29-36.

22. The Trauma Audit and Research Network (TARN). Available at: https:// www.tarn.ac.uk/Content.aspx?c=1895. Accessed September 28, 2013.

23. Hasler RM, Nüesch E, Jüni P, Bouamra O, Exadaktylos AK, Lecky F. Systolic blood pressure below $110 \mathrm{mmHg}$ is associated with increased mortality in penetrating major trauma patients: multicentre cohort study. Resuscitation. 2012;82(9):1202-1207.

24. Hasler RM, Nuesch E, Jüni P, Bouamra O, Exadaktylos AK, Lecky F. Systolic blood pressure below $110 \mathrm{~mm} \mathrm{Hg}$ is associated with increased mortality in blunt major trauma patients: multicentre cohort study. Resuscitation. 2011;82(9):1202-1207.

25. Perel P, Prieto-Merino D, Shakur H, Clayton T, Lecky F, Bouamra O, Russel R, Faulkner M, Steyerberg EW, Roberts I. Predicting early death in patients with traumatic bleeding: development and validation of prognostic model. Br Med J. 2012;345:e5166.

26. Ross SE1, Leipold C, Terregino C, O'Malley KF. Efficacy of the motor component of the Glasgow Coma Scale in trauma triage. J Trauma. 1998; 45(1):42-44.

27. Vivien B, Yeguiayan JM, Le Manach Y, Bonithon-Kopp C, Mirek S, Garrique D, Freysz M, Riou B. The motor component does not convey all the mortality prediction capacity of the Glasgow Coma Scale in trauma patients. Am J Emerg Med. 2012;30(7):1032-1041. 